\title{
Empirical tests of evolutionary synthesis models
}

\author{
Jean Michel Gomes ${ }^{1,2}$ \& R. Cid Fernandes ${ }^{1}$ \\ ${ }^{1}$ Dpto. de Física - CFM - Universidade Federal de Santa Catarina, Florianópolis, SC, Brazil \\ ${ }^{2}$ GEPI, Observatoire de Paris, CNRS, Université Paris Diderot; Place Jules Janssen 92190 \\ Meudon, France
}

\begin{abstract}
Spectral synthesis of stellar populations has proven to be one of the most powerful methods to decompose the different mixtures of stellar contributions in galaxies, and applications of this technique routinely appear in the literature nowadays. Our group, for instance, the SEAGal (Semi Empirical Analysis of Galaxies) collaboration, has derived the star formation history of all galaxies in the SDSS with the STARLIGHT code, obtaining various results of astrophysical interest. As any other fossil method, the results rely heavily on high spectral resolution evolutionary synthesis models. To test this model dependence we run STARLIGHT on samples of star-forming and passive galaxies from the SDSS using different sets of models.

We explore models using "Padova 1994" and modified "Padova" evolutionary tracks with a different receipt for the asymptotic giant branch phase, as well as different stellar libraries (STELIB versus MILES+Granada). We then compare derived properties such as mean age, mean metallicity, extinction, star-formation and chemical histories. Despite a broad brush agreement, systematic differences emerge from this comparison. The different evolutionary tracks used lead to essentially the same results, at least insofar as optical spectra are concerned. Different stellar libraries, on the other hand, have a much bigger impact. The newer models produce quantifiably better fits and eliminate some pathologies (like suspicious combinations of base elements, systematical spectral residuals in some windows, and, sometimes, negative extinction) of fits derived with STELIB-based models, but there are still some caveats. These empirical tests provide useful feedback for model makers.
\end{abstract}

Keywords. galaxies: evolution, galaxies: synthesis models, galaxies: stellar content.

\section{Introduction}

We will show a series of results obtained using different Simple Stellar Populations (SSP) in our semi-empirical synthesis method (Cid Fernandes et al. 2005), based on the following equation:

$$
\frac{M(\lambda)}{M\left(\lambda_{0}\right)}=\sum_{j=1}^{N_{\star}} x_{j}\left(\frac{L_{j}(\lambda)}{L_{j}\left(\lambda_{0}\right)}\right) r(\lambda) \otimes G\left(v_{\star}, \sigma_{\star}\right)
$$

where, $M(\lambda)$ is the model as a function of the wavelength, $L_{j}(\lambda)$ is the spectrum of a SSP, $\lambda_{0}$ is the normalization wavelength, $x_{j}$ is the fraction of light that each SSP will contribute to the total observed spectrum of a galaxy (total of $N_{\star}$ stellar populations), $r(\lambda)$ is the reddening term (modeled as due to an uniform dust screen, parameterized by $\left.A_{V}\right)$ and $G\left(v_{\star}, \sigma_{\star}\right)$ is the line-of-sight stellar velocity distribution. The match between the observed and modeled spectrum are done via statistical mechanics techniques using the minimization of the $\chi^{2}$ function. 


\section{SSP models and the data}

For comparison we have used distinct SSP models: (1) BC03 + STELIB - SSP with "Padova 1994" tracks and STELIB stellar library; (2) BC03 + MILESG - the same evolutionary tracks of (1), but using the stellar libraries of MILES and Granada; (3) CB07 + STELIB - preliminary models with different ingredients in the TP-AGB phase, but using the same library as (1); (4) CB07 + MILESG - the same library as (2) with the same ingredients as (3). To better understand these models see the references at the end of this work. Our samples consist of 1000 passives and 1000 star-forming galaxies selected randomly from the Main Galaxy Sample (Strauss et al. 2002) through a classical diagnostic diagram.

\section{Results}
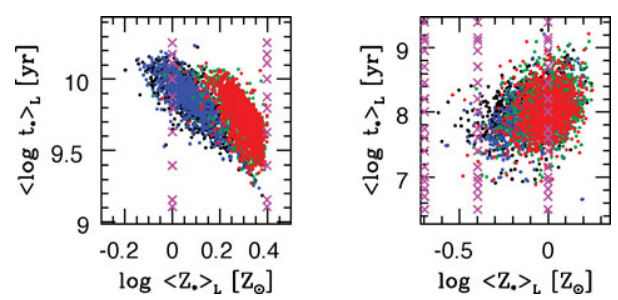

Figure 1. We have synthesized 1000 passives (left-panel) and 1000 star-forming galaxies (rightpanel). We show the mean ages and mean metallicities computed using: $\left\langle\log t_{\star}\right\rangle_{L}=\sum_{j}^{N_{\star}} x_{j} \log t_{j}$ and $\log \left\langle Z_{\star}\right\rangle_{L}=\log \left(\sum_{j}^{N_{\star}} x_{j} Z_{j}\right)$ for different SSP models. The colors stand for: black dots are BC03 + STELIB, blue dots are CB07 + STELIB, green dots are BC03 + MILESG and red dots are CB07 + MILESG models. The differences are for distinct stellar libraries, specifically for the passive galaxies and can reach 0.1 dex for $\left\langle\log t_{\star}\right\rangle_{L}$ and 0.2 dex for $\log \left\langle Z_{\star}\right\rangle_{L}$.
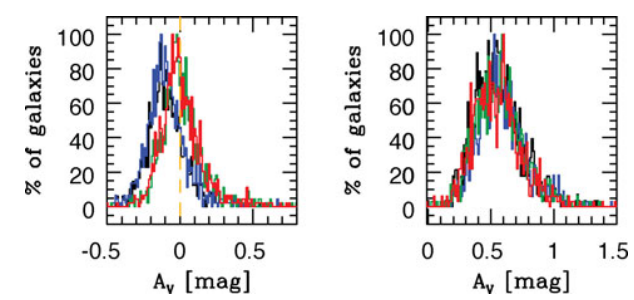

Figure 2. The extinctions derived using distinct SSP models for passives (left-panel) and starforming (right-panel). We found some pathologies with respect to models using the STELIB library when we analyze passive galaxies. The $\left\langle A_{V}\right\rangle$ are systematically negative, but are centered about zero for MILESG models. The colors follow the same criteria as figure 1 .

\section{References}

Bruzual, G. \& Charlot, S. 2003, MNRAS, 344, 1000

Le Borgne, J.-F., Bruzual, G., Pell, R., Lanon, A., Rocca-Volmerange, B., Sanahuja, B., Schaerer, D., Soubiran, C., \& Vlchez-Gmez, R. 2003, A\&A, 402, 433

Cid Fernandes, R., Mateus, A., Sodré, L., Stasińska, G., \& Gomes, J. M. 2005, MNRAS, 358, 363

Sánchez-Blázquez, P. Peletier, R. F. Jiménez-Vicente, J. Cardiel, N. Cenarro, A. J. FalcónBarroso, J. Gorgas, J. Selam, S., \& Vazdekis, A. 2006, MNRAS, 371, 703 